\title{
PRODUÇÃO DA ARARUTA 'COMUM' PROVENIENTE DE TRÊS TIPOS DE PROPÁGULOS
}

\author{
Production of 'Comum' arrowroot obtained from three types of propagules
}

\author{
Néstor Antonio Heredia Zárate ${ }^{1}$ Maria do Carmo Vieira ${ }^{1}$
}

\begin{abstract}
RESUMO
O trabalho foi desenvolvido em Dourados-MS, no período de 2/10/2003 a 21/8/2004, em Latossolo Vermelho distroférrico, textura argilosa. O objetivo foi avaliar a capacidade produtiva da araruta 'Comum', utilizando-se três tipos de propágulos. Os propágulos eram formados por pedaços de rizomas, com seis gemas, tomados da base ( $3,7 \mathrm{~g}$ a $9,0 \mathrm{~g}$; média de $6,0 \mathrm{~g})$, da parte média ( $7,1 \mathrm{~g}$ a $17,7 \mathrm{~g}$; média de $11,9 \mathrm{~g}$ ) ou da ponta do rizoma (10,2 g a 23,5 g; média de 16,7 g). Os tratamentos foram arranjados no delineamento experimental de blocos casualizados, com oito repetições. A colheita foi efetuada aos 324 dias após o plantio. A massa fresca das folhas, na época da colheita, não diferiu estatisticamente entre os tipos de propágulos. Houve aumentos significativos de $1,40 \mathrm{t} \mathrm{ha}^{-1} ; 8,88 \mathrm{tha}^{-1} ; 6,52 \mathrm{t} \mathrm{ha}^{-1} ; 1,13 \mathrm{tha}^{-1} \mathrm{e} 1,23 \mathrm{tha}^{-1}$, respectivamente, nas massas frescas de raiz, de rizomas totais, dos rizomas grandes, médios e pequenos, das plantas originadas de propágulos da parte média dos rizomas, em relação às das bases. O número de rizomas por hectare e a massa unitária dos rizomas foram influenciados significativamente pelos tipos de propágulos, com diferenças variáveis dentro de cada tamanho de rizoma produzido. Os rizomas de tamanho médio e pequeno foram influenciados significativamente pelo tipo de propágulo, sendo maiores nas plantas originadas a partir da parte média dos rizomas, em relação às das plantas originadas a partir das pontas ou das bases. Nas condições em que foi conduzido o experimento, concluiu-se que, para o plantio da araruta 'Comum', deve-se recomendar o uso de propágulos formados por pedaços da parte média ou das pontas dos rizomas, com seis gemas.
\end{abstract}

Termos para indexação: Maranta arundinaceae L., mudas, rizomas, produtividade.

\begin{abstract}
This work was carried out in Dourados-MS, from October $2^{\text {nd }}, 2003$, to August 21 $1^{\text {st }}, 2004$, in a Dystrorthox Soil, clay texture. The objective was to evaluate the productivity capacity of 'Comum' industrial Arrowroot using three types of propagules. Propagules were pieces of rhizomes with six buds, got from the base $(3.7 \mathrm{~g}$ to $9.0 \mathrm{~g}$; average of $6.0 \mathrm{~g}$ ) from medium part (7.1g to $17.7 \mathrm{~g}$; average $11.9 \mathrm{~g}$ ) or from the rhizome apex $(10.2 \mathrm{~g}$ to $23.5 \mathrm{~g}$; average of $16.7 \mathrm{~g})$. Treatments were arranged in a randomized experimental block design with eight replications. Harvest was done on 324 days after planting. Fresh mass of leaves, on harvest date, did not differ

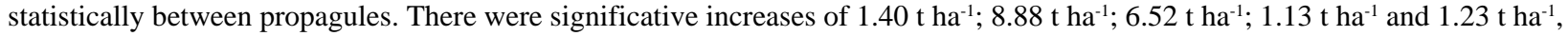
respectively, for fresh masses of roots of total rhizomes, of big, medium and small rhizomes from plants which originated from propagules of medium part of rhizomes, in relation to the bases. Number of rhizomes per hectare and unitary mass of rhizomes were influenced significantly by types of propagules, with variable differences inside each size of produced rhizome. Rhizomes of medium and small sizes were influenced by type of propagules, which were higher in plants originated from medium part of rhizomes in relation to those originated from apexes or bases. In the conditions wich the experiment was carried out, it was conclude that, for 'Comum" arrowroot planting, it is recommended the use of propagules originated from pieces of medium part or the apex of the rhizomes with six buds.
\end{abstract}

Index terms: Maranta arundinaceae L., sprouts, rhizomes, productivity.

\section{(Recebido para publicação em 8 de outubro de 2004 e aprovado em 13 de abril de 2005)}

\section{INTRODUÇÃO}

A araruta (Maranta arundinaceae L.) pertence à família Marantaceae, e seus rizomas contêm uma fécula que se presta a todas as combinações com a água e o leite, conseqüentemente à confecção de inúmeros pratos como biscoitos, bolos, cremes e doces, sendo recomendada, sobretudo, para convalescentes e crianças de 6 a 8 meses. O rizoma fresco contém, conforme a idade da planta, mais de $20 \%$ de amido (PEREIRA et al., 1999), além de substâncias acres, que os índios aplicam, de maneira tópica, contra os ferimentos provocados por flechas, envenenadas ou não, e como antídoto nas picadas de insetos ou nas mordidas de cobras peçonhentas (MONTEIRO \& PERESSIN, 2002).

A araruta teve seu centro de origem, provavelmente, no continente sul-americano, encontrando-se em forma nativa nas matas venezuelanas, posteriormente foi exportada às Ilhas Barbados, Jamaica e outras regiões do Caribe. Da araruta, três são as cultivares de importância no

1UFMS-DCA, Bolsista de Produtividade em Pesquisa do CNPq - Cx. P. 533 - 79804-970 Dourados, MS - nheredia@ceud.ufms.br. 
Brasil: Comum, Creoula e Banana, sendo que as duas primeiras são predominantes, com a primeira sendo a mais difundida comercialmente. As plantas da cultivar Comum são as que produzem fécula de melhor qualidade; são de porte baixo (mais ou menos $60 \mathrm{~cm}$ de altura); seus rizomas são claros, cônico-alongados ou em forma de fuso, cobertos por escamas e que atingem até $30 \mathrm{~cm}$ de comprimento, dependendo da qualidade do solo, embora o tamanho normal varie de 10 a $25 \mathrm{~cm}$. Também se caracteriza pelo pouco ou nenhum florescimento nas condições tropicais. $\mathrm{Na}$ 'Creoula', originária das Antilhas (Ilhas Barbados e Saint Vincent), a planta é de porte alto (superior a 1,0 m); produz rizomas na superfície do solo, em touceiras, que precisam ser lavados várias vezes para perder a camada superficial, caso contrário produzem fécula negra e de baixa qualidade. Apresenta florescimento abundante nas condições tropicais, sem, contudo, haver formação de frutos e sementes (LEONEL \& CEREDA, 2002; MONTEIRO $\&$ PERESSIN, 2002).

Monteiro \& Peressin (2002) relatam que a produção mundial da araruta é pequena e que tem-se informações de cultivos comerciais realizados em Barbados e Saint Vincent, no Caribe. Também citam que o IBGE, em 1997, relatou que a produção brasileira em 1996 foi de 1.141 toneladas, com valor estimado em R $\$ 283.565,15$, sendo que São Paulo contribuiu com 54 toneladas. A importância atual da araruta está muito relacionada com as características culinárias peculiares do seu amido. Como o preço alcançado pelo seu amido, no mercado internacional, é mais elevado que os similares, é grande o interesse dos industriais do setor na sua produção (MONTEIRO \& PERESSIN, 2002). Silva (1996) cita que a industrialização da araruta e da batatadoce, para obtenção de fécula, poderia racionalizar as indústrias de mandioca nos seus períodos de moagem, evitando a ociosidade da entressafra.

Um dos fatores que tem limitado a expansão de culturas propagadas vegetativamente, segundo Sediyama \& Casali (1997), é a falta de material de plantio e por isso é recomendado o bom aproveitamento das mudas. Vários aspectos das mudas ainda não foram estudados e, se o foram, os resultados ainda não são conclusivos. Em razão disso, muitas informações disponíveis originam-se da experiência de produtores e de extensionistas. Mas, há que ter cuidado, porque o tipo e a qualidade do material de plantio determinam diferenças na velocidade de enraizamento, crescimento e, conseqüentemente, produção e extensão do ciclo vegetativo.

Para as culturas de ciclo longo, como é o caso da araruta, é muito importante se conhecer o tipo e o tamanho da muda, assim como a forma que deve ser plantada, e, portanto, há necessidade de estabelecer o mais rápido a população final desejada. Na mandioquinha-salsa (Arracacia xanthorrhiza Bancroft), que apresenta ciclo de 7 a 12 meses (VIEIRA et al., 1996), para a multiplicação utilizam-se rebentos ou mudas (SEDIYAMA \& CASALI, 1997; VIEIRA, 1995) e no inhame (Dioscorea sp.), com ciclo de 9 a 12 meses, utilizam-se rizomas e/ou tubérculos (HEREDIA ZÁRATE et al., 2003). Monteiro \& Peressin (1997) estudando o efeito de dois tamanhos de rizomassemente (grande $=5 \mathrm{~g}$ e pequeno $=2 \mathrm{~g}$ ) na propagação do mangarito (Xanthosoma mafaffa Schott), observaram que, em termos de produção, os rizomas-semente do tipo grande superaram os pequenos.

Nas regiões tropicais, segundo Monteiro \& Peressin (2002), o plantio de araruta deve ser feito no período de meados de agosto a meados de outubro. $\mathrm{O}$ plantio é feito em sulcos ou em covas rasas $(0,10 \mathrm{~m}$ de profundidade) espaçados na linha de 0,70 a $0,80 \mathrm{~m}$ e entre plantas de 0,30 a $0,40 \mathrm{~m}$. Para a propagação e plantio comercial, podem ser utilizados tanto os rizomas inteiros, com massa média de $60 \mathrm{~g}$, como a parte basal (parte fina) de rizomas graúdos, entre 50 a $100 \mathrm{~g}$. A quantidade de rizomas-semente, necessária para o plantio está em torno de 2,0 a 3,0 $\mathrm{t} \mathrm{ha}^{-1}$ (LAURA et al., 2000; MONTEIRO \& PERESSIN, 2002).

Laura et al. (2000) estudaram a propagação da araruta usando duas classes de rizomas (classe $\mathrm{I}=$ rizomas com massa menor que 5,0 $\mathrm{g}$ e classe II= rizomas com massa entre 5,1 e 15,0 g) sob imersão em ácido indolbutírico (doses de 0, 150 e $300 \mathrm{mg} \mathrm{L}^{-1}$ ). Concluíram que a massa do rizoma é extremamente importante para o crescimento da parte aérea, das raízes e dos rizomas novos, sendo recomendados os rizomas com mais de 5,0 g, sem o uso do ácido indolbutírico.

Este trabalho teve como objetivo avaliar a capacidade produtiva da araruta 'Comum', utilizando-se três tipos de propágulos, formados por diferentes partes dos rizomas, em Dourados-MS.

\section{MATERIAL E MÉTODOS}

O trabalho foi desenvolvido no Horto de Plantas Medicinais da Universidade Federal de Mato Grosso do Sul - UFMS, em Dourados - MS, no período de 2/10/2003 a 21/8/2004. O município de Dourados situa-se em latitude

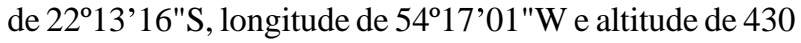
m. O clima da região, segundo a classificação de Köppen, citado por Mato Grosso do Sul (1990), é Mesotérmico Úmido; do tipo Cwa, com temperaturas e precipitações médias anuais variando de $20^{\circ}$ a $24^{\circ} \mathrm{C}$ e de 1250 a $1500 \mathrm{~mm}$,

Ciênc. agrotec., Lavras, v. 29, n. 5, p. 995-1000, set./out., 2005 
respectivamente. O solo é do tipo Latossolo Vermelho distroférrico, textura argilosa, com teores de M.O. $=3,2 \mathrm{~g} \mathrm{dm}^{-}$ 3; $\mathrm{P}=6,0 \mathrm{mg} \mathrm{dm}^{-3} ; \mathrm{K}=3,0 ; \mathrm{Ca}=39,7$ e $\mathrm{Mg}=28,2 \mathrm{mmol}_{\mathrm{c}} \mathrm{dm}^{-3} \mathrm{e}$ $\mathrm{pH} \mathrm{em} \mathrm{H}_{2} 0=6,1$.

Utilizando-se três tipos de mudas, formadas por um pedaço de rizoma, com seis gemas, tomados da base ( $3,7 \mathrm{~g}$ a $9,0 \mathrm{~g}$; média de $6,0 \mathrm{~g}$ ), da parte média $(7,1 \mathrm{~g}$ a $17,7 \mathrm{~g}$; média de $11,9 \mathrm{~g}$ ) ou da ponta do rizoma (10,2 $\mathrm{g}$ a $23,5 \mathrm{~g}$; média de $16,7 \mathrm{~g}$ ), foi feita a propagação da araruta 'Comum'. $\mathrm{O}$ delineamento experimental utilizado foi o de blocos casualizados, com oito repetições. Cada parcela teve $1,8 \mathrm{~m}^{2}$ (1,50 m de largura e 1,20 m de comprimento) e área útil de $1,20 \mathrm{~m}^{2}$ (1,00 m de largura x 1,20 m de comprimento), com dezoito plantas, arranjadas em fileira tripla, com espaçamentos de $0,333 \mathrm{~m}$ entre fileiras simples e $0,20 \mathrm{~m}$ entre plantas, perfazendo população de 99.000 plantas ha' ${ }^{-1}$.

O solo para o experimento foi preparado de forma convencional, constituindo-se de aração, gradagem e levantamento de canteiros com rotoencanteirador. Não efetuaram-se adubação nem calagem. No dia do plantio, as mudas foram selecionadas e classificadas por tipo, cortadas horizontalmente a cada seis gemas e pesadas. $\mathrm{O}$ plantio consistiu na abertura de sulcos de plantio de 0,05 $\mathrm{m}$ de largura e $0,05 \mathrm{~m}$ de profundidade, onde colocaram-se as mudas em posição vertical, com as gemas para acima, e posterior cobertura com terra. As irrigações foram feitas por aspersão, de forma a manter o solo "sempre úmido", o que induziu a turnos de rega a cada dois dias na fase de brotação, até as plantas apresentarem em torno de $0,20 \mathrm{~m}$ de altura e daí, em diante, as irrigações foram feitas duas vezes por semana. Foram feitas cinco capinas manuais.

A colheita efetuou-se aos 324 dias após o plantio, quando as plantas apresentavam mais de $50 \%$ de senescência da parte foliar, época em que avaliaram-se as produções de massa fresca de raízes, folhas e rizomas. Os rizomas foram classificados nos tamanhos grande, médio e pequeno. Também foram determinados os números de rizomas por tamanho e as características de diâmetro, comprimento e massa dos rizomas de cada tamanho. Os dados obtidos foram submetidos à análise de variância e quando verificou-se significância pelo teste $\mathrm{F}$, aplicou-se o teste de Tukey, até $5 \%$ de probabilidade.

\section{RESULTADOS E DISCUSSÃO}

A massa fresca das folhas, na época da colheita, não diferiu estatisticamente entre os tipos de propágulos (Tabela 1). Esses resultados sugerem que as plantas alcançaram a maturidade e iniciaram o processo de senescência das folhas mais velhas (VIEIRA et al., 1998) com translocação dos fotoassimilados de reserva para os órgãos armazenadores (VIEIRA, 1995), que no caso da araruta são os rizomas, independente do tipo de propágulo.

$\mathrm{O}$ fato de as maiores produções de massa fresca de rizomas terem sido obtidas de plantas de araruta provenientes das mudas de tamanho meio e ponta, respectivamente, ou seja, das mudas maiores (Tabela 1), permite deduzir que a quantidade de reserva presente na muda é importante fator relacionado ao crescimento e produção (HEREDIA ZÁRATE et al., 2003) da araruta. Essa dedução mostra relação com o aumento significativo de $1,40 \mathrm{t} \mathrm{ha}^{-1}$ de massa fresca de raízes e de $8,88 \mathrm{t} \mathrm{ha}^{-1} ; 6,52 \mathrm{t}$ $\mathrm{ha}^{-1} ; 1,13 \mathrm{t} \mathrm{ha}^{-1}$ e $1,23 \mathrm{t} \mathrm{ha}^{-1}$, na de rizomas total e nas massas frescas dos rizomas grandes, médios e pequenos, respectivamente, das plantas originadas a partir da parte média dos rizomas $(11,9 \mathrm{~g})$, em relação às das bases dos rizomas $(6,0 \mathrm{~g})$. Esses resultados mostram-se coerentes com Laura et al. (2000) que, estudando a propagação da araruta com duas classes de rizomas (classe $\mathrm{I}=$ rizomas com peso menor que 5,0 g e classe $\mathrm{II}=$ rizomas com peso entre 5,1 e $15,0 \mathrm{~g}$ ) sob imersão em ácido indolbutírico (doses de 0,150 e $300 \mathrm{mg} \mathrm{L}^{-1}$ ), concluíram que no cultivo da araruta, o peso do rizoma é extremamente importante para o crescimento da parte aérea, raízes e rizomas novos, sendo recomendados os rizomas com mais de 5,0 g. Também mostram relação com os resultados obtidos por Vieira et al. (1998), que estudaram a propagação da mandioquinha-salsa 'Amarela de Carandaí' utilizando mudas de tamanho grande (26,1 g), médio $(14,2 \mathrm{~g})$, pequeno $(8,5 \mathrm{~g})$ e muito pequeno $(5,5 \mathrm{~g}) \mathrm{e}$ constataram que plantas provenientes de mudas médias tiveram maiores produções de raízes comerciais do que mudas pequenas.

Os aumentos não-significativos de $0,89 \mathrm{t} \mathrm{ha}^{-1} \mathrm{de}$ massa fresca de raízes e de 4,30; 3,37; 0,73 e 0,20 t ha ${ }^{-1}$ nas massas frescas de rizomas total e dos rizomas grandes, médios e pequenos, respectivamente, das plantas originadas a partir da parte média dos rizomas, em relação às das plantas originadas a partir das pontas dos rizomas, mostraram relação inversa, mas não-significativa, com a massa fresca de folhas na época de colheita $\left(11,26 \mathrm{t} \mathrm{ha}^{-1}\right.$ para as das pontas e 9,82 $\mathrm{t} \mathrm{ha}^{-1}$ para as da parte média) e relação direta com as produções de raízes (Tabela 1). Resultados análogos obtidos por Heredia Zárate et al. (2002, 2003) mostram que embora a planta inteira seja autotrófica, seus órgãos individuais dependem uns dos outros, para obter nutrientes e fotoassimilados. Também, Casali et al. (1984) trabalhando com mandioquinha-salsa, observaram que as plantas que apresentaram crescimento exuberante 
não produziram muito bem, deduzindo que essas plantas podem ter retardado a maturidade e o início do processo de senescência das folhas mais velhas (VIEIRA et al., 1998), com diminuição da translocação dos fotoassimilados de reserva para os órgãos armazenadores (VIEIRA, 1995). Hashad et al. (1956) observaram em cultivo de taro (Colocasia esculenta (L.) Schott) que os fotoassimilados são translocados dos limbos para os pecíolos e, finalmente, para os rizomas.

O número e a massa unitária dos rizomas por unidade de área foram influenciados significativamente pelos tipos de propágulos, com diferenças variáveis dentro de cada tamanho de rizoma produzido (Tabela 2). Esses resultados são coerentes com a hipótese de que a partição dos fotoassimilados é função do genótipo e das relações fonte-dreno, em que a eficiência de conversão fotossintética, dentre outros fatores, pode ser alterada pelas condições de solo, clima e estádio fisiológico da cultura (TAIZ \& ZEIGER, 2004).
Os rizomas de tamanho grande apresentaram valores médios de diâmetro e comprimento semelhantes e, não influenciados pelo tipo de propágulo. Já, os rizomas de tamanho médio e pequeno foram influenciados significativamente pelo tipo de propágulo, sendo maiores nas plantas originadas a partir da parte média dos rizomas, em relação às das plantas originadas a partir das pontas ou das bases (Tabela 3). Esses resultados permitem observar de que esses caracteres são intrínsecos da espécie (HEREDIA ZÁRATE, 1988), do clone utilizado e da forma de condução da cultura (HEREDIA ZÁRATE et al., 1999). Isso porque, a capacidade de as plantas destinarem, prioritariamente, recursos para a reprodução, sobrevivência, desenvolvimento, crescimento e defesa é uma das características adaptativas importantes definidas pelo princípio de alocação de fotoassimilados proposto por Cody (1966), citado por Fancelli \& Dourado Neto (1996).

TABELA 1 - Produção de massa fresca de folha, raiz e rizoma de plantas da araruta 'Comum', em função de três tipos de propágulos. Dourados, UFMS, 2003-2004.

\begin{tabular}{lrccccc}
\hline & \multicolumn{5}{c}{ Produção de massa fresca $\left(\mathbf{t ~ h a}^{\mathbf{- 1}}\right)$} \\
\cline { 2 - 6 } $\begin{array}{l}\text { Tipo de } \\
\text { mudas }\end{array}$ & \multirow{2}{*}{ folha } & raiz & grande & médio & pequeno & total \\
\cline { 2 - 7 } & & $3,19 \mathrm{~b}$ & $8,22 \mathrm{~b}$ & $2,91 \mathrm{~b}$ & $2,91 \mathrm{~b}$ & $14,04 \mathrm{~b}$ \\
\hline Base & 8,70 & $3,70 \mathrm{ab}$ & $11,37 \mathrm{ab}$ & $3,31 \mathrm{ab}$ & $3,94 \mathrm{a}$ & $18,62 \mathrm{ab}$ \\
Ponta & 11,26 & $4,59 \mathrm{a}$ & $14,74 \mathrm{a}$ & $4,04 \mathrm{a}$ & $4,14 \mathrm{a}$ & $22,92 \mathrm{a}$ \\
Meio & 9,82 & 22,00 & 35,11 & 16,67 & 10,89 & 26,64 \\
\hline C.V. & 15,81 & &
\end{tabular}

Médias seguidas pelas mesmas letras nas colunas não diferem, pelo teste de Tukey, a 5\% de probabilidade.

TABELA 2 - Número e massa fresca unitária de três tamanhos de rizomas produzidos por plantas da araruta 'Comum', em função de três tipos de propágulos. Dourados, UFMS, 2003-2004.

\begin{tabular}{|c|c|c|c|c|c|c|}
\hline \multirow{3}{*}{$\begin{array}{l}\text { Tipo de } \\
\text { mudas }\end{array}$} & \multicolumn{6}{|c|}{ Tamanho de rizoma } \\
\hline & \multicolumn{2}{|c|}{ grande } & \multicolumn{2}{|c|}{ médio } & \multicolumn{2}{|c|}{ pequeno } \\
\hline & $\begin{array}{c}\text { número } \\
\left(\text { mil ha }^{-1}\right)\end{array}$ & $\begin{array}{c}\text { massa } \\
\text { unitária }(\mathrm{g})\end{array}$ & $\begin{array}{l}\text { número } \\
\left(\text { mil ha }^{-1}\right)\end{array}$ & $\begin{array}{c}\text { massa } \\
\text { unitária }(g)\end{array}$ & $\begin{array}{l}\text { número } \\
\left(\text { mil ha }^{-1}\right)\end{array}$ & $\begin{array}{c}\text { massa } \\
\text { unitária }(\mathrm{g})\end{array}$ \\
\hline Base & $106,57 \mathrm{~b}$ & $71,18 \mathrm{~b}$ & $91,74 \mathrm{~b}$ & $33,02 \quad \mathrm{c}$ & $219,62 \mathrm{~b}$ & $13,36 \mathrm{~b}$ \\
\hline Ponta & $140,85 \mathrm{ab}$ & $77,69 a$ & $85,25 \mathrm{~b}$ & $38,37 \mathrm{a}$ & $283,56 a$ & $14,19 \mathrm{ab}$ \\
\hline Meio & $180,63 \mathrm{a}$ & $80,87 \mathrm{a}$ & $116,76 \mathrm{a}$ & $35,28 \mathrm{~b}$ & $253,73 \mathrm{ab}$ & $15,91 \mathrm{a}$ \\
\hline C.V. & 40,66 & 5,06 & 12,40 & 3,99 & 11,86 & 8,01 \\
\hline
\end{tabular}

Médias seguidas pelas mesmas letras nas colunas não diferem, pelo teste de Tukey, a 5\% de probabilidade.

Ciênc. agrotec., Lavras, v. 29, n. 5, p. 995-1000, set./out., 2005 
TABELA 3 - Diâmetro e comprimento de três tamanhos de rizomas produzidos por plantas da araruta 'Comum', em função de três tipos de propágulos. Dourados, UFMS, 2003-2004.

\begin{tabular}{|c|c|c|c|c|c|c|}
\hline \multirow{3}{*}{$\begin{array}{l}\text { Tipo de } \\
\text { mudas }\end{array}$} & \multicolumn{6}{|c|}{ Tamanho de rizoma } \\
\hline & \multicolumn{2}{|c|}{ grande } & \multicolumn{2}{|c|}{ médio } & \multicolumn{2}{|c|}{ pequeno } \\
\hline & $\begin{array}{l}\text { diâmetro } \\
\text { (cm) }\end{array}$ & $\begin{array}{l}\text { comprimento } \\
\text { (cm) }\end{array}$ & $\begin{array}{l}\text { diâmetro } \\
(\mathrm{cm})\end{array}$ & $\begin{array}{c}\text { comprimento } \\
\text { (cm) }\end{array}$ & $\begin{array}{l}\text { diâmetro } \\
\text { (cm) }\end{array}$ & $\begin{array}{c}\text { comprimento } \\
(\mathbf{c m})\end{array}$ \\
\hline Base & 3,18 & 20,47 & $2,61 \mathrm{~b}$ & 14,53 & $2,13 \mathrm{~b}$ & $11,52 \mathrm{a}$ \\
\hline Ponta & 3,34 & 20,92 & $2,70 \mathrm{ab}$ & 14,44 & $2,19 \mathrm{~b}$ & $9,50 \mathrm{~b}$ \\
\hline Meio & 3,30 & 20,70 & $2,77 \mathrm{a}$ & 15,16 & $2,29 \mathrm{a}$ & $12,00 \mathrm{a}$ \\
\hline C.V. & 3,21 & 6,75 & 2,03 & 4,87 & 2,03 & 9,60 \\
\hline
\end{tabular}

Médias seguidas pelas mesmas letras nas colunas não diferem, pelo teste de Tukey, a 5\% de probabilidade.

\section{CONCLUSÃO}

Para o plantio da araruta 'Comum', deve-se recomendar o uso de propágulos formados por pedaços da parte média ou das pontas dos rizomas, com seis gemas.

\section{AGRADECIMENTOS}

Ao CNPq, pelas bolsas concedidas, e à FUNDECT$\mathrm{MS}$, pelos recursos financeiros.

\section{REFERÊNCIAS BIBLIOGRÁFICAS}

CASALI, V. W. D.; SEDIYAMA, M. A. N.; CAMPOS, J. P. Métodos culturais da mandioquinha-salsa. Informe Agropecuário, Belo Horizonte, v. 10, n. 120, p. 26-28, 1984.

FANCELLI, A. L.; DOURADO NETO, D. Milho: fisiologia da produção. In: SEMINÁRIO SOBRE FISIOLOGIA DA PRODUÇÃO E MANEJO DE ÁGUA E DE NUTRIENTES NA CULTURA DO MILHO DE ALTA PRODUTIVIDADE, 1996, Piracicaba. Palestras... Piracicaba: ESALQ/USPPOTAFÓS, 1996. p. 1-29.

HASHAD, M. N.; STINO, K. R.; EL-HINNAMY, S. I. Transformation and translocation of carbohidrates in taro plants during growth. Annals of Agricultural Sciences, Cairo, v. 1, n. 1, p. 261-267, 1956.

HEREDIA ZÁRATE, N. A. Curvas de crescimento de inhame (Colocasia esculenta (L.) Schott), considerando cinco populações, em solo seco e alagado. 1988. $95 \mathrm{f}$. Tese (Doutorado em Fitotecnia) - Universidade Federal de Viçosa, Viçosa, 1988.
HEREDIA ZÁRATE, N. A.; VIEIRA, M. C.; FACCO, R. C. Produção de clones de inhame em função do tamanho das mudas. Acta Scientiarum: Agronomy, Maringá, v. 25, n. 1, p. 183-186, 2003.

HEREDIA ZÁRATE, N. A.; VIEIRA, M. C.; GRIEP, R. Produção dos clones de cará Liso e Caramujo conduzidos em forma rasteira e tutorada. Horticultura Brasileira, Brasília, v. 17, n. 1, p. 45-48, 1999.

HEREDIA ZÁRATE, N. A.; VIEIRA, M. C.; MINUZZI, A. Brotação de seis tipos de mudas dos clones de inhame roxo e mimoso. Ciência e Agrotecnologia, Lavras, v. 26, n. 4, p. 699-704, 2002.

LAURA, V. A.; CHAVES, F. C. M.; QUIJANO, F. O. G.; CÂMARA, F. L. A. Brotação e particionamento de assimilados em rizomas de araruta: efeito do peso dos rizomas e da concentração de IBA. In: CONGRESSO BRASILEIRO DE OLERICULTURA, 40.; CONGRESSO IBERO-AMERICANO SOBRE UTILIZAÇÃO DE PLÁSTICO NA AGRICULTURA, 2.; SIMPÓSIO LATINO AMERICANO DE PRODUÇÃO DE PLANTAS MEDICINAIS, AROMÁTICAS E CONDIMENTARES, 1., 2000, São Pedro, SP. Horticultura Brasileira, Brasília, v. 18, p. 325-326, 2000.

LEONEL, M.; CEREDA, M. P. Caracterização físico-química de algumas tuberosas-amiláceas. Ciência e Tecnologia de Alimentos, Campinas, v. 22, n. 1, p. 65-69, 2002.

MATO GROSSO DO SUL. Secretaria de Planejamento e Coordenação Geral. Atlas multireferencial. Campo Grande, 1990. 28 p. 
MONTEIRO, D. A.; PERESSIN, V. A. Efeito do tamanho do rizoma-semente, da época e do local de plantio, na produção de rizomas de mangará. Bragantia, Campinas, v. 56, n. 1, p. 155-161, 1997.

MONTEIRO, D. A.; PERESSIN, V. A. Cultura da araruta. In: CEREDA, M. P. (Coord.). Agricultura: tuberosas amiláceas Latino Americanas. São Paulo: Fundação Cargill, 2002. v. 2, p. 440-447.

PEREIRA, J.; CIACCO, C. F.; VILELA, E. R.; TEIXEIRA, L. de S. Fermented starch in the biscuit manufacturing: alternative sources. Ciência e Tecnologia de Alimentos, São Paulo, v. 19, n. 2, p. 287-293, 1999. Disponível em: <http:/ 1/Www.scielo.br/scielo.php?script=sci_arttext\&pid=S0101 $20611999000200024 \& \operatorname{lng}=\mathrm{en} \& \mathrm{nrm}=\mathrm{iso}$. Acesso em: 2 set. 2004 .

SEDIYAMA, M. A. N.; CASALI, V. W. D. Propagação vegetativa da mandioquinha-salsa. Informe Agropecuário, Belo Horizonte, v. 19, n. 190, p. 24-27, 1997.

SILVA, J. R. B. Mandioca e outras raízes tropicais: uma base alimentar da humanidade no século XXI. In:
CONGRESSO LATINO AMERICANO DE RAÍZES TROPICAIS, 1.; CONGRESSO BRASILEIRO DE MANDIOCA, 9., 1996, São Pedro, SP. Palestras... São Pedro: CERAT, 1996. p. 12-15.

TAIZ, L.; ZEIGER, E. Fisiologia vegetal. 3. ed. Porto Alegre: Artmed, 2004. 720 p.

VIEIRA, M. C. Avaliação do crescimento e da produção de clones e efeito de resíduo orgânico e de fósforo em mandioquinha-salsa no Estado de Mato Grosso do Sul. 1995. 146 f. Tese (Doutorado em Fitotecnia) - Universidade Federal de Viçosa, Viçosa, 1995.

VIEIRA, M. C.; HEREDIA ZÁRATE, N. A.; SIQUEIRA, J. G. de; CASALI, V. W. D. Crescimento e produção de mandioquinha-salsa em função de características das mudas. Horticultura Brasileira, Brasília, v. 14, n. 1, p. 42-44, 1996.

VIEIRA, M. C.; HEREDIA ZÁRATE, N. A.; VITORINO, P. F. G. Produção de mandioquinha-salsa em resposta ao tamanho de mudas e populações. Horticultura Brasileira, Brasília, v. 16, n. 1, 1998. (Resumo 363).

Ciênc. agrotec., Lavras, v. 29, n. 5, p. 995-1000, set./out., 2005 